\title{
纳米多孔结构镍基复合膜电极的电化学法制备及其电容特性
}

\author{
孔德帅 ${ }^{1}$ 王建明 ${ }^{1, *}$ 皮欧阳 ${ }^{1} \quad$ 邵海波 $^{1} \quad$ 张鉴清 $^{1,2}$ \\ ('浙江大学化学系, 杭州 310027; 2中国科学院金属研究所, 腐蚀与防护国家重点实验室, 沈阳 110016)
}

\begin{abstract}
摘要: 通过对电沉积法得到的 $\mathrm{Ni}-\mathrm{Cu}$ 合金镀层进行电化学去合金化处理, 制备了纳米多孔结构金属镍膜. 采用 循环伏安法对多孔金属镍膜在 $1 \mathrm{~mol} \cdot \mathrm{L}^{-1} \mathrm{KOH}$ 溶液中进行阳极氧化处理, 获得了纳米多孔结构的镍基复合膜 电极. 应用扫描电子显微镜(SEM)、X射线衍射 (XRD)、X射线光电子能谱(XPS)和电化学技术对所制备的膜电 极的物理性质及噟电容特性进行了表征. SEM、XRD 和 XPS 的测试结果表明, 所制备的纳米多孔结构镍基复 合膜由 $\mathrm{Ni} 、 \mathrm{Ni}(\mathrm{OH})_{2}$ 和 $\mathrm{NiOOH}$ 组成. 电化学实验结果显示, 该复合膜在 $20 \mathrm{~A} \cdot \mathrm{g}^{-1}$ 的充放电电流密度下, 给出了 $578 \mathrm{~F} \cdot \mathrm{g}^{-1}$ 的初始比电容; 在 1000 次充放电循环后, 它的比电容值为 $544 \mathrm{~F} \cdot \mathrm{g}^{-1}$, 电容保持率为 $94 \%$. 纳米多孔 结构有利于 $\mathrm{KOH}$ 电解液的渗透, 从而促进反应物种在电极内部的传输; 纳米多孔的金属镍基体可以提高 $\mathrm{Ni}(\mathrm{OH})_{2}$ 膜的电子导电性; 纳米大小的 $\mathrm{Ni}(\mathrm{OH})_{2}$ 颗粒能够缩短质子的固相扩散路径. 上述因素是所制备的纳米多 孔结构镍基复合膜电极具有优异噟电容特性的主要原因.
\end{abstract}

关键词: 电化学电容器; 纳米多孔结构; 氢氧化镍; 去合金化; 比电容; 电沉积 中图分类号: 0646

\section{Electrochemical Fabrication and Pseudocapacitive Performance of a Porous Nanostructured Nickel-Based Complex Film Electrode}

\author{
KONG De-Shuai ${ }^{1} \quad$ WANG Jian-Ming ${ }^{1, *} \quad$ PI Ou-Yang ${ }^{1} \quad$ SHAO Hai-Bo ${ }^{1} \quad$ ZHANG Jian-Qing ${ }^{1,2}$ \\ ('Department of Chemistry, Zhejiang University, Hangzhou 310027, P. R. China; ${ }^{2}$ State Key Laboratory for Corrosion and \\ Protection of Metal, Institute of Metal Research, Chinese Academy of Sciences, Shenyang 110016, P. R. China)
}

\begin{abstract}
A porous nickel film was prepared by the selective anodic dissolution of copper from an electrodeposited $\mathrm{Ni}$-Cu alloy film. A porous nanostructured nickel-based complex film electrode was further fabricated by oxidizing the obtained porous nickel film using cyclic voltammetry in $1 \mathrm{~mol} \cdot \mathrm{L}^{-1} \mathrm{KOH}$ solution. The physical properties and pseudocapacitive performance of the as-prepared film electrodes were investigated by scanning electron microscopy (SEM), X-ray diffraction (XRD), X-ray photoelectron spectroscopy (XPS), and electrochemical techniques. The results of SEM, XRD, and XPS indicate that the obtained complex film electrode consists of $\mathrm{Ni}, \mathrm{Ni}(\mathrm{OH})_{2}$, and $\mathrm{NiOOH}$, and it has a porous nanostructure. The electrochemical experiments revealed that the as-prepared porous nanostructured nickel-based complex film electrode had a specific capacitance of $578 \mathrm{~F} \cdot \mathrm{g}^{-1}$ at a current density of $20 \mathrm{~A} \cdot \mathrm{g}^{-1}$ at the initial cycle and it gave a specific capacitance of $544 \mathrm{~F} \cdot \mathrm{g}^{-1}$ after 1000 cycles with a capacitance retention of $94 \%$. The nanoporous structure enhances the accessibility of the $\mathrm{KOH}$ electrolyte and promotes reactive species transport within the electrode. The nanoporous Ni substrate may improve the electronic conductivity of the thin $\mathrm{Ni}(\mathrm{OH})_{2}$ film at its surfaces. The nanosized $\mathrm{Ni}(\mathrm{OH})_{2}$ grains can shorten the proton diffusion pathways in the bulk of the solid nickel hydroxide. These factors are responsible for the superior pseudocapacitive performance of the porous nanostructured nickel-based complex film electrode.
\end{abstract}

Received: December 20, 2010; Revised: February 18, 2011; Published on Web: March 11, 2011.

"Corresponding author. Email: wjm@zju.edu.cn; Tel: +86-571-87951513.

The project was supported by the National Natural Science Foundation of China (50972128).

国家自然科学基金(50972128)资助项目

(C) Editorial office of Acta Physico-Chimica Sinica 
Key Words: Electrochemical capacitor; Nanoporous structure; Nickel hydroxide; Dealloying; Specific capacitance; Electroplating

\section{1 引言}

超级电容器又称电化学电容器, 是一种新型的 储能器件, 具有比功率高、可逆性好和循环寿命长等 特点. ${ }^{1-5}$ 在超级电容器金属氧化物材料中, $\mathrm{RuO}_{2}$ 具 有较高的比容量 $\left(720 \mathrm{~F} \cdot \mathrm{g}^{-1}\right)$ 和良好的循环稳定性, ${ }^{6,7}$ 然而昂贵的价格限制了它的商业化应用. 寻找具有 高比容量的廉价电极材料是电化学电容器领域的研 究重点之一, 主要集中在过渡金属氧化物/氢氧化物 如 $\mathrm{NiO} / \mathrm{Ni}(\mathrm{OH})_{2} 、{ }^{8,9} \mathrm{Co}_{3} \mathrm{O}_{4} / \mathrm{Co}(\mathrm{OH})_{2}{ }^{10,11}$ 和 $\mathrm{MnO}_{2}{ }^{12}$ 等.

$\mathrm{NiO} / \mathrm{Ni}(\mathrm{OH})_{2}$ 由于具有电极电位高、电化学容量 大与电化学循环稳定性好等优异特性, 被认为是很 有潜力的电化学电容器用电极材料. ${ }^{13-15}$ 为了提高电 极的电容性能, 人们已使用沉淀转化、 ${ }^{16}$ 溶胶-凝胶、 ${ }^{17}$ 磁控溅射、年电化学阴极/阳极沉积 ${ }^{9,19}$ 等方法制备了 氢氧化镍/氧化镍电极材料. 不同方法所制备材料的 电容性能不尽相同, 但其电容值大多为 50-350 F. $\mathrm{g}^{-1}$, 远低于相应材料的理论电容值 (在 $0.5 \mathrm{~V}$ 的电位 窗口内 $\mathrm{NiO}$ 和 $\mathrm{Ni}(\mathrm{OH})_{2}$ 的理论电容值分别为 2584 和 $\left.2082 \mathrm{~F} \cdot \mathrm{g}^{-1}\right)$; 而且电极的循环稳定性也有待于提高.

电极材料的性能与其比表面积和表面结构密切 相关, 具有较高比表面积和纳米多孔网状结构的电 极材料可以显示优异的电容特性. ${ }^{3.19}$ 在纳米多孔结 构电极的制备方法中, 电化学方法可在导电基体上 原位形成活性物质膜, 而且通过改变沉积电位/电流 及电解液组成等参数, 能够较方便地对膜的形貌和 微结构进行调控, 被认为是制备氢氧化镍膜电极较 好的方法. 920

本文通过对电沉积法得到的 $\mathrm{Ni}-\mathrm{Cu}$ 合金镀层进 行电化学去合金化处理, 制备了纳米多孔结构金属 镍膜; 采用循环伏安法对其进行阳极氧化处理, 获 得了纳米多孔结构的镍基复合膜电极. 应用循环伏 安和恒流充放电等方法测试了所制备电极的电容性 能.

\section{2 实验部分}

\section{1 仪器与试剂}

扫描电子显微镜 (SEM, SIRION-100, 美国 FEI 公司); X 射线衍射仪(D/Max 2550, 日本 Rigaku 公 司); X 射线光电子能谱仪( $5000 \mathrm{C}$, 美国 PHI 公司); 精 密电子天平(CP225D, 德国 Sartorius 公司); 电化学工
作站(CHI 660C, 上海辰华仪器公司). 所用试剂均为 分析纯, 溶液用去离子水配制.

\section{2 多孔镍膜与复合膜电极的制备}

多孔金属镍的制备采用一种完全电化学方法, 即首先在电极基体上阴极电沉积 $\mathrm{Ni}-\mathrm{Cu}$ 合金镀层, 之后通过去合金化法溶解金属铜, 从而得到多孔金 属镍电极. ${ }^{21} \mathrm{Ni}-\mathrm{Cu}$ 合金镀层的阴极电沉积在室温下 于三电极体系中进行, 不锈钢片 $\left(2.0 \mathrm{~cm}^{2}\right)$ 为工作电 极, $\mathrm{Pt}$ 和 $\mathrm{Ag} / \mathrm{AgCl}$ 分别作为对电极和参比电极. 沉积 液为 $1.0 \mathrm{~mol} \cdot \mathrm{L}^{-1} \mathrm{NiSO}_{4} 、 0.05 \mathrm{~mol} \cdot \mathrm{L}^{-1} \mathrm{CuSO}_{4}$ 和 0.5 $\mathrm{mol} \cdot \mathrm{L}^{-1} \mathrm{H}_{3} \mathrm{BO}_{3}$ 的混合溶液. 电沉积前不锈钢基体用 1200 目的砂纸打磨, 然后分别用丙酮和去离子水清 洗. 阴极电沉积在 $-0.8 \mathrm{~V}$ 的恒电位下进行, 沉积电量 为 $2.0 \mathrm{C}$. 阴极沉积之后, 在相同的三电极体系中, 施 加 $0.5 \mathrm{~V}$ 的阳极电位, 溶解金属铜, 截止电流密度为 $15.0 \mu \mathrm{A} \cdot \mathrm{cm}^{-2}$. 通过前述电化学过程, 即可得到多孔 金属镍膜.

多孔金属镍膜的电化学阳极氧化使用三电极体 系, 多孔金属镍膜、 $\mathrm{Pt}$ 和 $\mathrm{Ag} / \mathrm{AgCl}$ 分别作为工作电 极、对电极和参比电极. 电解液为 $1.0 \mathrm{~mol} \cdot \mathrm{L}^{-1} \mathrm{KOH}$ 溶液. 采用循环伏安法在 $0-0.5 \mathrm{~V}$ 的电位范围内以 $20 \mathrm{mV} \cdot \mathrm{s}^{-1}$ 的扫描速率对多孔金属镍膜进行循环阳 极氧化处理, 循环次数为 50 , 可制得镍基复合膜电 极.

\section{3 电化学性能测试}

所制备的镍基复合膜电极的循环伏安和恒流充 放电测试采用与多孔金属镍膜的电化学阳极氧化相 同的三电极体系.

\section{3 结果与讨论}

\section{1 多孔镍膜和镍基复合膜的表面形貌与结构}

阴极电沉积法制备的 Ni-Cu 合金镀层为柱形颗 粒状沉积层(图略), 与文献 ${ }^{21}$ 报道的结果一致. 文献 ${ }^{21,22}$ 结果表明, 在 $\mathrm{Ni}-\mathrm{Cu}$ 合金沉积层中存在着相分离现 象, 即富铜相存在于柱状颗粒的中心, 而富镍相则位 于富铜相的四周. 在 $0.5 \mathrm{~V}$ 的阳极电位下, 铜核发生 阳极溶解, 而镍壳因钝化得以保留, 于是形成了多孔 镍膜. ${ }^{21}$ 图 1 给出了多孔镍膜在氧化处理前后的 SEM 照片, 由图 1a可见, 所制得的多孔镍膜是由孔径为 80-200 nm 的中空镍管组成的. 图 1b 显示, 采用循 

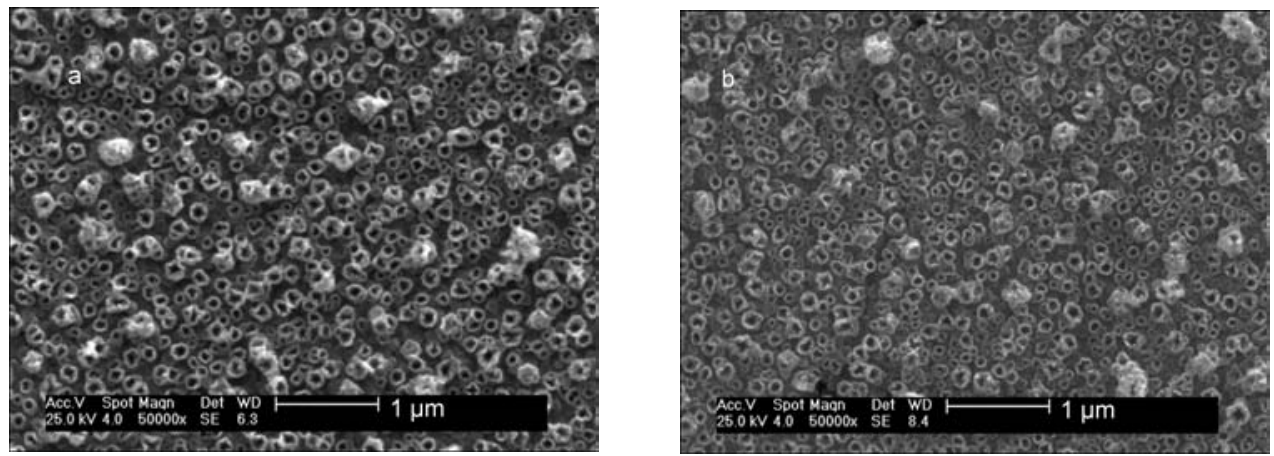

图 1 多孔镍膜在氧化处理前(a)后(b)的 SEM照片

Fig.1 SEM images of porous nickel films before (a) and after (b) oxidation

环伏安法氧化处理后, 多孔膜的孔径略变小; 而明 显变暗的 SEM 照片表明多孔膜的表面导电性降低. 上述结果可能意味着 $\mathrm{Ni}(\mathrm{OH})_{2} / \mathrm{NiOOH}$ 膜原位生长 在多孔镍膜的表面.

图 2 为多孔镍膜在循环伏安法氧化前后的 XRD 图谱. 未经氧化处理的多孔电极的XRD图谱(图 2a) 在 $2 \theta$ 为 $44.52^{\circ} 、 51.88^{\circ}$ 和 $76.32^{\circ}$ 处出现较强峰, 可以 分别归于金属镍的(111)、(200)和(220)晶面, ${ }^{22}$ 证实了 对电沉积合金层进行阳极去合金化处理得到的多孔 膜由金属镍组成. 循环伏安法氧化处理后多孔镍膜 的所有衍射峰均明显弱化, 只有(111)晶面的强度还 相对较高. 这说明经氧化处理后金属镍表面可能形 成了氧化膜, 而且不同晶面的氧化程度不同, (111) 晶面的氧化程度相对较低. ${ }^{23}$ 但在图 $2 \mathrm{~b}$ 中未发现较 强的新衍射峰出现, 意味着形成的氧化膜较薄或结 晶度较差. 为了鉴别镍表面的氧化膜, 对氧化后的样 品进行了 XPS 测试, 结果示于图 3. 在 856-858 eV 之间较宽的 Ni $2 p_{3 / 2}$ XPS 峰表明氧化膜主要由 $\mathrm{Ni}(\mathrm{OH})_{2}$ 和 $\mathrm{NiOOH}$ 组成. ${ }^{24,25}$ 上述结果表明, 多孔镍膜 在循环伏安法氧化过程中发生如下反应:

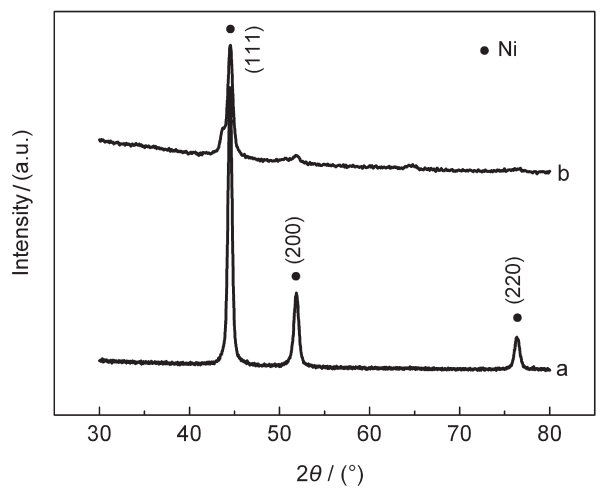

图 2 多孔镍膜在氧化处理前(a)后(b)的XRD 图谱

Fig.2 XRD patterns of porous nickel films before (a) and after (b) oxidation

$$
\begin{aligned}
& \mathrm{Ni}+2 \mathrm{OH}^{-} \rightarrow \mathrm{Ni}(\mathrm{OH})_{2}+2 \mathrm{e}^{-} \\
& \mathrm{Ni}(\mathrm{OH})_{2}+\mathrm{OH}^{-} \rightleftharpoons \mathrm{NiOOH}+\mathrm{H}_{2} \mathrm{O}+\mathrm{e}^{-}
\end{aligned}
$$

在所应用的氧化电位窗口 $(0.0-0.5 \mathrm{~V})$ 内, 反应(1)是 不可逆的, 而反应(2)则是可逆的. 因此, 通过对纳米 多孔金属镍基体的循环伏安法氧化处理,得到了纳 米多孔结构的 $\mathrm{NiOOH} / \mathrm{Ni}(\mathrm{OH})_{2} / \mathrm{Ni}$ 复合膜电极. 应该 指出的是, 通过控制电位扫描的范围及速率等条件 可以对电极表面膜中 $\mathrm{Ni}(\mathrm{OH})_{2}$ 和 $\mathrm{NiOOH}$ 的比例进行 调控.

\section{2 纳米多孔结构镍基复合膜电极的电容特性}

图 4 显示了纳米多孔结构复合膜电极在不同扫 描速率下的循环伏安曲线. 在所测试的循环伏安曲 线中均出现了一对较强的氧化/还原峰, 对应于 $\mathrm{Ni}^{2+}$ 和 $\mathrm{Ni}^{3+}$ 的相互转化过程(反应(2)). ${ }^{9}$ 由图 4 可见, 在不 同扫描速率下氧化与还原峰均具有良好的对称性; 随着扫描速率的增加, 氧化/还原峰电流几乎线性增 大, 而相应的峰电位变化很小. 这表明所制备的复合 膜电极具有良好的电化学活性和可逆性. 循环伏安 结果表明, 复合膜电极的比电容主要来源于氧化还 原反应(反应(2))法拉第噟电容的贡献.

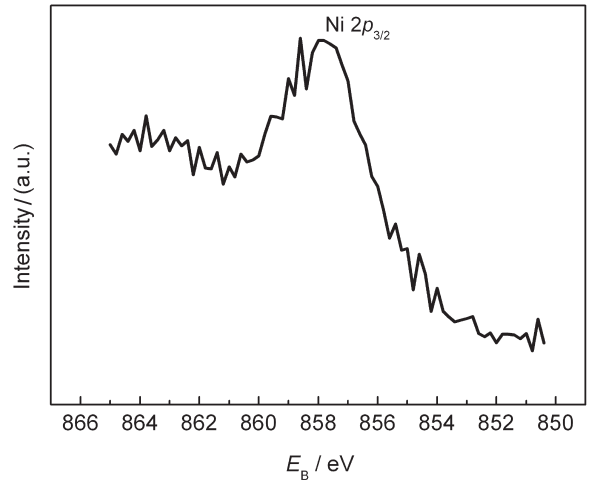

图 3 经氧化处理的多孔镍膜在 Ni $2 p$ 区域的 XPS 图谱

Fig.3 XPS spectrum of the oxidized porous nickel film for $\mathrm{Ni} 2 p$ region 


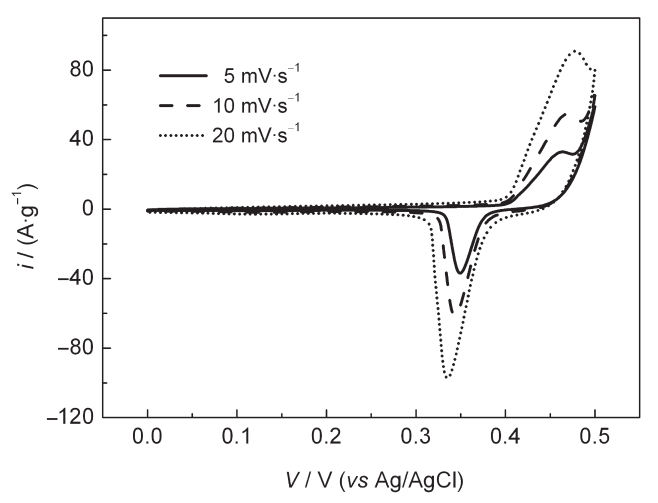

图 4 复合膜电极在不同扫描速率下的循环伏安曲线

Fig.4 CV curves of the complex film electrode at different scan rates

图 5A 为所制备的纳米多孔结构复合膜电极在 不同电流密度下的放电曲线. 所测试的放电曲线均 显示了明显的放电平台, 起源于 $\mathrm{NiOOH}$ 还原为 $\mathrm{Ni}(\mathrm{OH})_{2}$ 的电极反应. 该结果进一步证实了复合膜电 极的噟电容特性. 由放电曲线可以计算电极的比电 容值, 公式如下: ${ }^{26}$

$$
C=\frac{i \cdot \Delta t}{m \cdot \Delta V}
$$

式中, $i$ 为放电电流密度, $\Delta t$ 为放电时间, $\Delta V$ 为放电 电压范围, $m$ 为电极材料的质量(在本文中为所沉积 的 $\mathrm{Ni} 、 \mathrm{Ni}(\mathrm{OH})_{2}$ 和 $\mathrm{NiOOH}$ 的总质量). 在 20 和 $50 \mathrm{~A}$. $\mathrm{g}^{-1}$ 的放电电流密度下, 所制备的复合膜电极在首次 放电中分别给出了 578 和 $372 \mathrm{~F} \cdot \mathrm{g}^{-1}$ 的比容量, 显示 了优异的容量与功率特性. 图 $5 \mathrm{~B}$ 显示了在 $20 \mathrm{~A} \cdot \mathrm{g}^{-1}$ 的充放电电流密度下复合膜电极的电化学循环性 能. 在初始的 200 次充放电循环中, 复合膜电极的比 电容略有降低, 之后基本保持不变. 1000 次充放电 循环后, 复合膜电极的比电容为 $544 \mathrm{~F} \cdot \mathrm{g}^{-1}$, 电容保 持率为 $94 \%$, 显示了优异的电化学循环稳定性. 应该 指出的是, 与目前大多数文献 ${ }^{9,16-20,26}$ 报道的结果相 比, 我们所制备的纳米多孔结构复合膜电极显示了 较高的比电容、良好的功率特性和明显改进的电化 学循环稳定性.

纳米结构复合膜电极具有高的比表面积, 可以 为电极表面法拉第反应提供较多的活性位; 多孔结 构能够改善 $\mathrm{KOH}$ 电解液在电极内部的传输, 增加电 极有效反应面积; 纳米多孔金属镍基体的存在可以 有效提高复合膜电极的电子导电性, 降低电极极 化; 而纳米大小的 $\mathrm{Ni}(\mathrm{OH})_{2} / \mathrm{NiOOH}$ 颗粒, 可以降低 固相质子扩散的路径, 从而降低扩散极化. ${ }^{5,27}$ 上述几 个因素是所制备的纳米多孔结构复合膜电极具有明
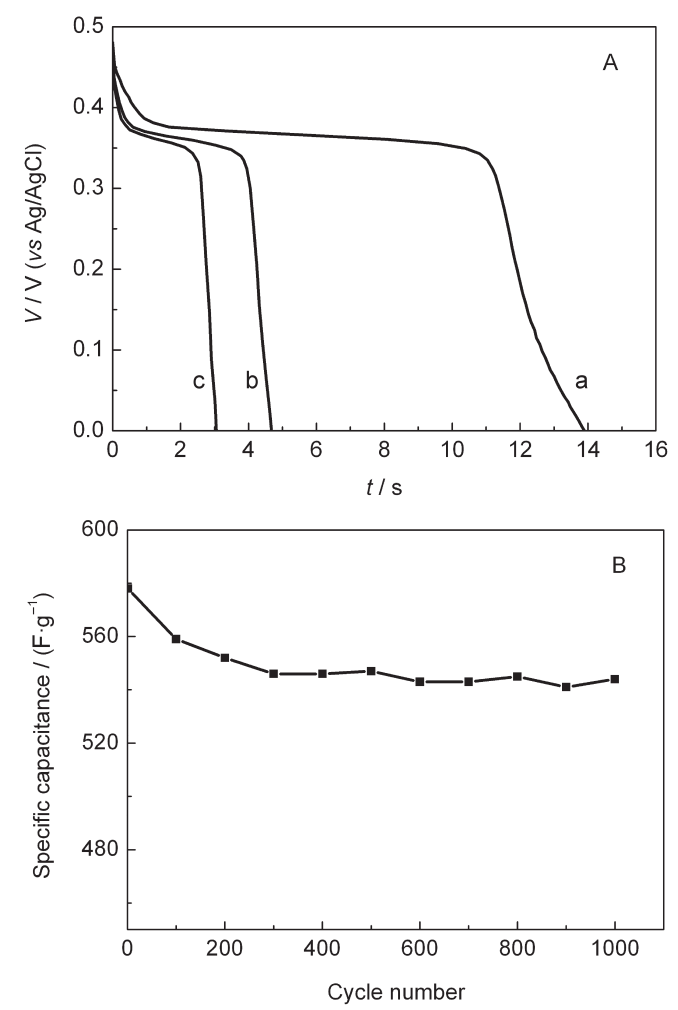

图 5 复合膜电极在不同电流密度下的放电曲线(A)和在 $20 \mathrm{~A} \cdot \mathrm{g}^{-1}$ 的电流密度下的循环性能(B)

Fig.5 Galvanostatic discharge curves of the complex film electrode at different current densities (A) and cyclic performance of the electrode at $20 \mathrm{~A} \cdot \mathrm{g}^{-1}$ (B) $i /\left(\mathrm{A} \cdot \mathrm{g}^{-1}\right):$ (a) 20 , (b) 40 , (c) 50

显改进的噟电容性能的主要原因. 应该说明的是, 我 们也曾以金属镍片为基体, 进行了与多孔镍膜相同 的氧化处理, 发现所制备的复合电极具有很差的电 容性能. 这进一步证实了前述结论.

\section{4 结 论}

通过对电沉积法制得的 $\mathrm{Ni}-\mathrm{Cu}$ 合金膜进行电化 学去合金化处理, 制备了由孔径为 80-200 nm 的中 空镍管组成的有序结构金属镍膜. 采用循环伏安法 对多孔金属镍膜在 $1 \mathrm{~mol} \cdot \mathrm{L}^{-1}$ 的 $\mathrm{KOH}$ 溶液中进行阳 极氧化处理, 得到了由 $\mathrm{Ni} 、 \mathrm{Ni}(\mathrm{OH})_{2}$ 和 $\mathrm{NiOOH}$ 组成的 纳米多孔结构的镍基复合膜电极. 在 $20 \mathrm{~A} \cdot \mathrm{g}^{-1}$ 的充 放电电流密度下, 所制得的纳米多孔结构镍基复合 膜电极在首次和第 1000 次充放电循环中分别显示 了 578 和 $544 \mathrm{~F} \cdot \mathrm{g}^{-1}$ 的比电容, 电容保持率为 $94 \%$. 纳 米多孔结构镍基复合膜电极的大比表面积、纳米多 孔结构、高电子导电性与纳米大小的活性粒子是其 显示优异赝电容性能的主要原因. 


\section{References}

(1) Chmiola, J.; Yushin, G.; Gogotsi, Y.; Portet, C.; Simon, P.; Taberna, P. L. Science 2006, 313, 1760.

(2) Arico, A. S.; Bruce, P.; Scrosati, B.; Tarascon, J. M.; Schalkwijk, W. V. Nat. Mater. 2005, 4, 366.

(3) Simon, P.; Gogotsi, Y. Nat. Mater. 2008, 7, 845.

(4) Miller, J. R.; Simon, P. Science 2008, 321, 651.

(5) Wu, M. S.; Huang, Y. A.; Yang, C. H. J. Electrochem. Soc. 2008, 155, A798.

(6) Zheng, J. P.; Cygan, P. J.; Jow, T. R. J. Electrochem. Soc. 1995, $142,2699$.

(7) Sugimoto, W.; Iwata, H.; Yasunaga, Y.; Murakami, Y.; Takasu, Y. Angew. Chem. Int. Edit. 2003, 42, 4092.

(8) Wu, M. S.; Huang, C. Y.; Lin, K. H. J. Power Sources 2009, $186,557$.

(9) Zhao, D. D.; Bao, S. J.; Zhou, W. J.; Li, H. L. Electrochem. Commun. 2007, 9, 869.

(10) Kong, L. B.; Lang, J. W.; Liu, M.; Luo, Y. C.; Kang, L. J. Power Sources 2009, 194, 1194.

(11) Zeng, W. W.; Huang, K. L.; Yang, Y. P.; Liu, S. Q.; Liu, R. S. Acta Phys. -Chim. Sin. 2008, 24, 263. [曾雯雯, 黄可龙, 杨幼 平, 刘素琴, 刘人生. 物理化学学报, 2008, 24, 263.]

(12) Hu, J.; Yuan, A. B.; Wang, Y. Q.; Wang, X. L. Acta Phys. -Chim. Sin. 2009, 25, 987. [胡 洁, 袁安保, 王玉芹, 王秀玲. 物理化 学学报, 2009, 25, 987.]
(13) Jeevanandam, P.; Koltypin, Y.; Gedanken, A. Nano Lett. 2001, $1,263$.

(14) Srinivasan, V.; Weidner, J. W. J. Electrochem. Soc. 2000, 147, 880.

(15) He, J. J.; Lindström, H.; Hagfeldt, A.; Lindquist, S. E. J. Phys. Chem. B 1999, 103, 8940.

(16) Liu, K. C.; Anderson, M. A. J. Electrochem. Soc. 1996, 143, 124.

(17) Wang, Y. G.; Xia, Y. Y. Electrochim. Acta 2006, 51, 3223.

(18) Lee, S. H.; Tracy, C. E.; Pitts, J. R. Electrochem. Solid State Lett. 2004, 7, A299.

(19) Zhao, D. D.; Zhou, W. J.; Li, H. L. Chem. Mat. 2007, 19, 3882.

(20) Wu, M. S.; Wang, M. J. Electrochem. Solid State Lett. 2010, 13, A1.

(21) Chang, J. K.; Hsu, S. H.; Sun, I. W.; Tsai, W. T. J. Phys. Chem. C 2008, 112, 1371.

(22) Sun, L.; Chien, C. L.; Searson, P. C. Chem. Mat. 2004, 16, 3125.

(23) Medway, S. L.; Lucas, C. A.; Kowal, A.; Nichols, R. J.; Johnson, D. J. Electroanal. Chem. 2006, 587, 172.

(24) Park, K. W.; Choi, J. H.; Kwon, B. K.; Lee, S. A.; Sung, Y. E. J. Phys. Chem. B 2002, 106, 1869.

(25) Hoflund, G. B.; Epling, W. S. Chem. Mat. 1998, 10, 50.

(26) Hosogai, S.; Tsutsumi, H. J. Power Sources 2009, 194, 1213.

(27) Wu, M. S.; Huang, Y. A.; Jow, J. J.; Yang, W. D.; Hsieh, C. Y.; Tsai, H. M. Int. J. Hydrog. Energy 2008, 33, 2921. 